\title{
Correlation of ocular and systemic factors with the presence of serous retinal detachment in diabetic macular edema
}

\begin{abstract}
Purpose: To study the systemic and ocular correlation of serous macular detachment (SRD) in patients with Type 2 Diabetes Mellitus with diabetic macular edema (DME).

Methods: This prospective study included 100 consecutive patients (both males and females) of type 2 diabetes, aged 30 years and above with DME in at least one eye. All patients had a detailed history recorded including duration of diabetes, treatment received, comorbidities and baseline systemic investigations. Slit lamp biomicroscopy, fundus fluorescein angiography and optical coherence tomography (OCT) were performed for all patients to characterize DME.

Results: Out of the 100 patients, 77 patients had bilateral and 23 cases had unilateral DME. Based on the presence or absence of SRD on OCT, patients were divided into 2 groups: (Group 1: DME with SRD and Group 2: DME without SRD). There was no correlation of any systemic factors with presence of SRD. Ocular factors like grade of retinopathy, central macular thickness, and combination of diffuse and cystoid DME and cystoid pattern of DME on OCT were found to be significantly associated with SRD in DME. On multivariate analysis, higher central macular thickness and cystoid pattern of DME on OCT were found to be the most significant predictors of serous macular detachment in DME.

Conclusion: Our study could not find correlation of any systemic factor with serous macular detachment in diabetic macular edema. However, ocular factors like severe NPDR, higher Central macular thickness, combination of diffuse and cystoid macular edema on FFA and cystoid pattern of DME on OCT were found to be significantly associated with $\mathrm{SRD}$ in DME.
\end{abstract}

Volume 7 Issue 6 - 2017

\author{
Mangat Ram Dogra, Kanika Aggarwal, \\ Gaurav Sanghi,Vishali Gupta, Ramandeep \\ Singh, Amod Gupta \\ Department of Ophthalmology, Advanced Eye Center, Post \\ Graduate Institute of Medical Education and Research \\ (PGIMER), India
}

Correspondence: Mangat Ram Dogra, Professor, Advanced Eye Center, Post Graduate Institute of Medical Education and Research (PGIMER), Chandigarh, India,Tel +9I I72 2756 I I I Email kanika2kI@yahoo.co.in

Received:September 16, 2017 | Published: November 22, 2017
Abbreviations: SRD: serous macular detachment; DME: diabetic macular edema; OCT: optical coherence tomography; LDL: low-density lipoprotein; HDL: high-density lipoprotein; VLDL: very low-density lipoprotein; IOP: intraocular pressure; NPDR: non-proliferative diabetic retinopathy; PDR: proliferative diabetic retinopathy; CMT: central macular thickness; PCV: packed cell volume; FBS: fasting blood sugar; PPBS: postprandial blood sugar; RPE: retinal pigment epithelium

\section{Introduction}

Diabetic macular edema (DME) is diagnosed clinically by stereoscopic slit lamp biomicroscopy examination using preferably a contact lens for fundus evaluation. However, this method lacks the ability to quantify the edema and has a limited sensitivity and specificity in diagnosing early and mild cases. ${ }^{1}$ Optical Coherence Tomography (OCT) is a newer modality that helps in the objective assessment of DME. OCT allows not only the qualitative diagnosis of DME but also the quantitative assessment of the edema. ${ }^{2-4}$ Otani et al. ${ }^{5,6}$ were the first to describe three distinct patterns of DME on the basis of OCT findings. These included sponge-like retinal swelling, cystoid macular edema and serous macular detachment (SRD). Diffuse retinal thickening and cystoid macular edema can be detected on slit-lamp biomicroscopy and/or fluorescein angiography.? However, SRD is often undetected by these methods in majority of the cases. OCT seems to be particularly useful for detecting SRD, a feature that is consistent with macular edema but that is not easily seen on biomicroscopy. ${ }^{8}$ It is only after the advent of OCT that this entity has been highlighted as a significant finding in many cases of DME leading to decreased visual acuity.

Various risk factors for DME have been identified. In younger onset diabetics with the age at diagnosis less than 30 years, DME is associated with longer duration of diabetes, presence of proteinuria, diuretic use, male gender and higher glycosylated haemoglobin. ${ }^{9}$ While in older onset diabetic patients, the presence of DME is associated with longer duration of diabetes, higher blood pressure, insulin use, higher glycosylated haemoglobin, presence of proteinuria, hyperlipidemia and anemia. ${ }^{10-12}$ Drugs like thiazolidinediones, mainly pioglitazone and rosiglitazone, are known to cause fluid retention and peripheral edema. Such fluid retention with glitazones can lead to worsening of DME. ${ }^{13}$ However, it is not known whether the above risks factors are variable for the different morphological patterns of DME. SRD is an infrequently studied pattern of DME which may be associated with various such risk factors. Hence this prospective study was planned to identify any ocular/systemic risk factors associated with the development of SRD in patients with DME.

\section{Materials and methods}

The prospective study included 100 consecutive patients (both males and females) of type 2 diabetes mellitus, aged 30 years and above with DME in at least one eye. The study was approved by the institute ethics committee and all the patients signed a written informed consent. The study adhered to the tenets of Declaration of Helsinki. All patients had detailed history recorded including duration of the disease, treatment received including details of the drugs and 
their dosages, history of any co-morbidity such as hypertension, renal, cardiac, neurological or peripheral vascular disease. All patients underwent the following baseline investigations: haemoglobin, glycosylated haemoglobin ( $\mathrm{HbA1c}$ ), systolic and diastolic blood pressure (two readings taken two to three minutes apart in the right arm in sitting position), blood urea, serum creatinine, spot urine albumin and total lipid profile including serum cholesterol, triglyceride, lowdensity lipoprotein (LDL), high-density lipoprotein (HDL) and very low-density lipoprotein (VLDL).

Ocular examination included the recording of the best-corrected visual acuity (BCVA) using Snellen's chart, intraocular pressure (IOP) on Goldmann applanation tonometry, slit-lamp biomicroscopy and fundus examination with $+90 \mathrm{D}$ lens, central 50-degrees fundus photograph and fundus fluorescein angiogram (Carl Zeiss Meditec, Dublin, CA, USA) and examination on high-definition spectraldomain OCT (CirrusTM OCT, Zeiss Meditec, Dublin, CA, USA). The fundus photographs were graded according to the severity of retinopathy into mild, moderate, severe, very severe non-proliferative diabetic retinopathy (NPDR) or proliferative diabetic retinopathy (PDR) based on the standard ETDRS classification. ${ }^{14}$ All OCT measurements were performed following pharmacological dilatation of the pupil. The OCT examination comprised 5-line raster scans and macular cube $512 \times 128$ combo for all patients. The central macular thickness (CMT) was measured and graded for the pattern of DME i.e. diffuse retinal swelling, cystoid macular edema or SRD. On the basis of OCT findings, the patients were divided into 2 groups:

Group 1: Patients with CSME with serous retinal detachment in at least one eye

Group 2: Patients with CSME without serous retinal detachment in either eye

The two groups were compared on the basis of various factors such as duration of diabetes, type of treatment (insulin/oral hypoglycaemic agents), blood pressure (systolic and diastolic), drugs for hypertension, presence/absence of microvascular and macrovascular complications of diabetes such as nephropathy, neuropathy, coronary artery disease, peripheral vascular disease, diabetic foot, among others; laboratory parameters such as glycosylated haemoglobin (HbA1c), haemoglobin, packed cell volume (PCV), fasting blood sugar (FBS), Postprandial blood sugar (PPBS), blood urea, serum creatinine, lipid profile, spot urine albumin (if macro-albuminuria was present then 24 hour urinary protein was obtained), best-corrected visual acuity, severity of retinopathy, pattern of diabetic macular edema on OCT and CMT. Statistical analysis was performed using Graph Pad. ${ }^{\circledR}$ The quantitative variables among the two groups were compared using Mann-Whitney U test. Qualitative data was compared using Chi-square test. Various ocular and systemic factors associated with DME and SRD were analyzed using univariate/multivariate analysis. A $p$ value of $<0.05$ was considered as statistically significant For the purpose of analysis of the systemic factors such as comorbidities, the various parameters were compared for the patients in the two groups whereas for analysing ocular factors such as visual acuity and pattern of DME on FFA and OCT were compared between the eyes in the two groups.

\section{Results}

Based on the presence/absence of serous macular detachment on OCT, the patients were grouped as: It is better for this classification to mention the number of eyes with SRD not the number of patients because although systemic factors can affect one eye before the other yet ocular factors are restricted to one eye. For example you can say that patient number one with renal impairment had SRD in one eye, this makes renal impairment an important factor in induction of SRD because both eyes are nearly never parallel but you cannot say that patient number one with severe NPDR OU has SRD in the left eye only because in this latter case the (severe NPDR) will be considered as a less effective factor in induction of SRD when counting the number of eyes not the number of patients. Group I: Included 30 patients (42 eyes) with CSME and serous macular detachment in at least one eye. Group II: Included 70 patients (135 eyes) with CSME without serous macular detachment in either eye.

There were a total of 100 patients ( 40 females and 60 males) with a median age of 56years. In Group I, there were 10 females and 20 males with a median age of 57 years (IQ range 51.5-62years) and a total of 42 eyes. In Group II, there were 30 females and 40 males with a median age of 56 years (IQ range 50.75-61.0years) and a total of 135 eyes. 5eyes in this group were excluded as they did not fulfil the inclusion criteria of presence of DME. The median age at onset of diabetes in Group 1 was 46.5 years (IQ range 39.75 - 52.5 years). The median age at onset of diabetes in Group 2 was $44.5 y e a r s$ (IQ range 38.75-50 years) which was not statistically significant using Mann Whitney test $(\mathrm{P}=0.159)$. The median duration of diabetes in Group 1 was 10 years (IQ range 7.5-15years) and in Group 2 was 11.5 (IQ range 8-15years) (Figures 1-4).

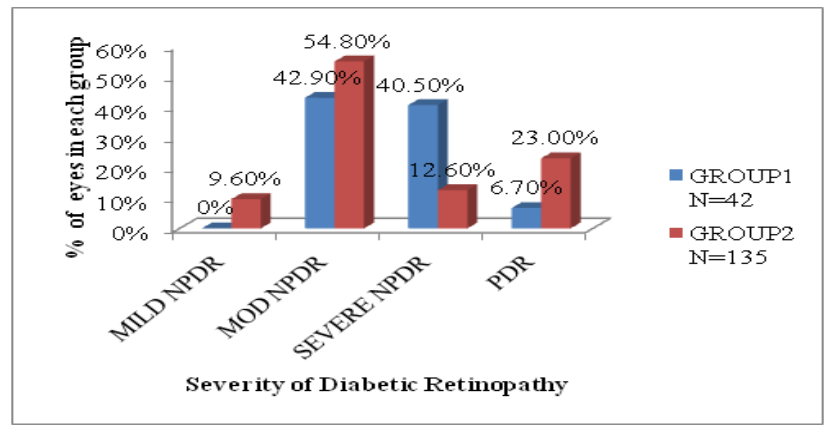

Figure I Severity of diabetic retinopathy.

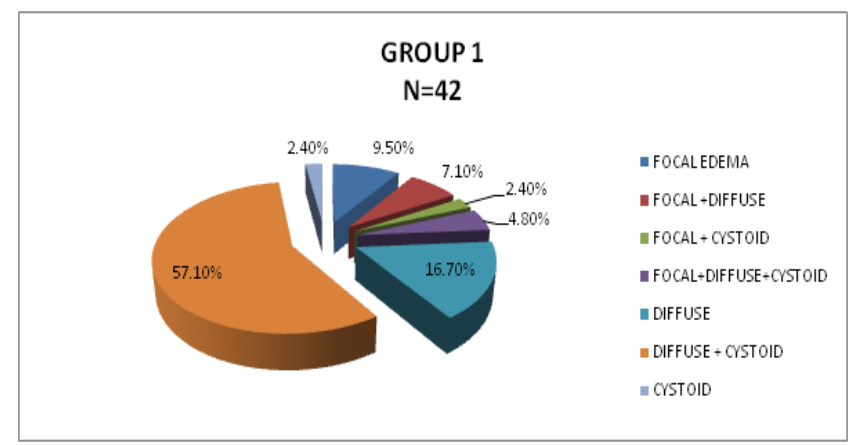

\section{Figure 2 Group I $\mathrm{N}=42$.}

The difference in the duration of diabetes between the two groups was statistically significant as analysed by Mann-Whitney test $(p=0.02)$. There was no statistically significant difference between the two groups in any of the drugs that the patients were receiving for diabetes. 16 patients (53.3\%), in Group 1 and 44 patients $(62.9 \%)$ in Group 2 had hypertension. There was no statistically significant difference between the two groups as analysed by Chi-square test 
( $\mathrm{P}=0.373$ ). The median duration of hypertension was 2years (IQ range 0.25-3 years) in Group 1 and 3years (IQ range 1.0-6.5years) in Group 2 . The difference between the two groups was not statistically significant as analysed by Mann- Whitney test $(p=0.07)$. The number of patients on calcium channel blockers were significantly higher in Group 2 as analysed by Chi-square test $(\mathrm{P}=0.04)$. The median systolic B.P was $135 \mathrm{mmHg}$ (range $120-150 \mathrm{mmHg}$ ) in group 1 and $130 \mathrm{mmHg}$ (range 127.5-140 $\mathrm{mmHg}$ ) in Group 2. The median diastolic B.P was $90 \mathrm{mmHg}$ (range $80-90 \mathrm{mmHg}$ ) in Group 1 and $90 \mathrm{mmHg}(80-90$ $\mathrm{mmHg}$ ) in Group 2. There was no statistically significant difference between the 2 groups in the prevalence of any comorbidity like CAD, nephropathy, neuropathy, etc. The 2 groups were comparable for weight, height and BMI.

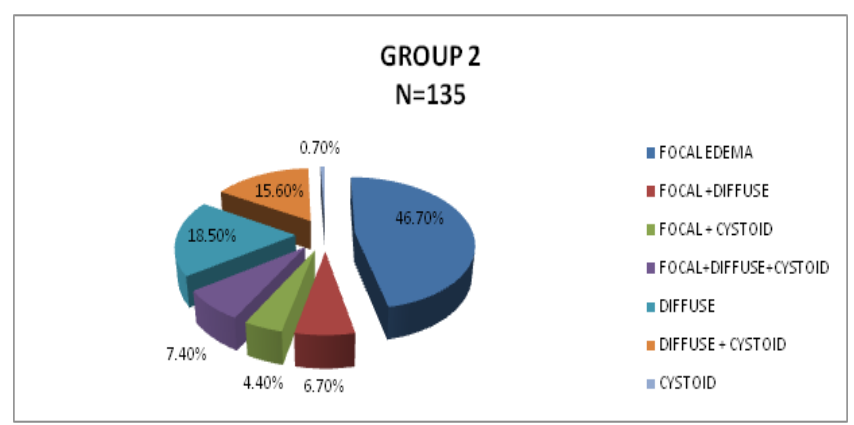

Figure 3 Group $2 \mathrm{~N}=\mid 35$.

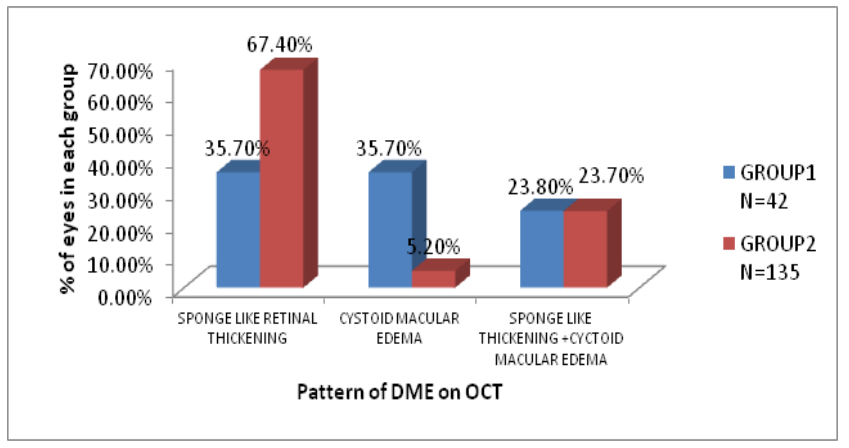

Figure 4 Pattern of DME on OCT.

None of the biochemical parameters showed any statistically significant difference between the two groups as analysed by the Mann Whitney test. The median LOGMAR visual acuity in both groups was 0.48 . Hence, there was no statistically significant difference in the visual acuity of eyes with or without serous retinal detachment. Group 1 consisted of no eyes with mild NPDR while Group 2 consisted of 13 eyes $(9.6 \%)$ with mild NPDR. Group 1 consisted of 18 eyes $(42.9 \%)$ with moderate NPDR while Group 2 consisted of 74 eyes (54.8\%) with moderate NPDR. Both the groups had 17 eyes $(40.5 \%$ and $12.6 \%$ respectively) with severe NPDR. Group 1 had 7 eyes (16.7\%) with PDR while Group 2 had 31 eyes (23\%) with PDR. On comparing the severity of diabetic retinopathy in both groups using Chi-square test, the number of eyes with severe NPDR is significantly more in Group 1 as compared to Group 2 (P .001).

Group 1 had focal macular edema in 4 eyes (9.5\%), diffuse macular edema in 7 eyes $(16.7 \%)$, cystoid macular edema in 1 eye $(2.4 \%)$, both focal and diffuse macular edema in 3 eyes (7.1\%), focal with cystoid macular edema in 1 eye $(2.4 \%)$, diffuse with cystoid macular edema in 24 eyes $(57.1 \%)$ and all three patterns of macular edema in 2 eyes $(4.8 \%)$.
Group 2 had focal macular edema in 63 eyes (46.7\%), diffuse macular edema in 25 eyes (18.5\%), cystoid macular edema in 1 eye $(0.7 \%)$, both focal and diffuse macular edema in 9 eyes $(6.7 \%)$, focal with cystoid macular edema in 6 eyes (4.4\%), diffuse with cystoid macular edema in $21(15.6 \%)$ eyes and all three patterns of macular edema coexisted in $10(7.4 \%)$ eyes.

Using Chi-square test for analysis, the fluorescein angiographic pattern of focal macular edema was found to be significantly more common in Group 2 eyes as compared to Group $1(p<0.001)$. The combination of diffuse and cystoid macular edema was significantly more common in Group 1 compared to Group 2 ( $p<0.001)$. The difference between the 2 groups as analysed by Mann-Whitney test was statistically significant $(p<0.001)$. Three main patterns of diabetic macular edema which were identified on OCT were:

a. Sponge like diffuse retinal swelling

b. Cystoid macular edema

c. Serous macular detachment

d. Combination of the above patterns

Group 1 had sponge like retinal swelling with serous macular detachment in 15 eyes (35.7\%) and cystoid macular edema with serous macular detachment also in 15 eyes (35.7\%). A combination of sponge like retinal swelling, cystoid macular edema and serous macular detachment was found in 10 eyes (23.8\%). Two patients had no specific pattern associated with serous macular detachment.

Group 2 had sponge like retinal swelling in 91 eyes 67.4\%), combination of sponge like swelling and cystoid macular edema in 34 eyes $(23.7 \%)$, cystoid macular edema alone in 7 eyes $(5.2 \%)$. Two eyes $(1.4 \%)$ in group 2 with a combination of sponge like retinal swelling and cystoid macular edema had associated vitreomacular traction/taut posterior hyaloid. Three patients had a normal foveal contour despite increased central macular thickness. Cystoid pattern of macular edema was present in a total of 25 eyes (59.5\%) in Group 1 and in 41 eyes $(30.3 \%)$ in Group 2. The difference between the two groups was statistically significant as analysed by Chi-square test $(\mathrm{P}<0.001)$. Since only two systemic variables (duration of diabetes and treatment with calcium channel blockers) were significant on univariate analysis, they were not entered into a multivariate regression analysis. On entering the significant ocular factors into a multivariate regression analysis, Central macular thickness and cystoid pattern of DME on OCT came out as most significant risk factors associated with serous macular detachment in DME (Table 1).

Table I The difference between the two groups

\begin{tabular}{lccl}
\hline & $\begin{array}{l}\text { Group I } \\
\text { N=42 }\end{array}$ & $\begin{array}{l}\text { Group 2 } \\
\text { N=I35 }\end{array}$ & P Value \\
\hline $\begin{array}{l}\text { Sponge Like Retinal } \\
\begin{array}{l}\text { Thickening } \\
\text { Cystoid Macular }\end{array}\end{array}$ & I5 (35.7\%) & 9I (67.4\%) & 0.037 \\
$\begin{array}{l}\text { Edema } \\
\text { Sponge Like }\end{array}$ & $75.7 \%)$ & $7(5.2 \%)$ & $<0.00$ I \\
$\begin{array}{l}\text { Thickening + Cystoid I0 (23.8\%) } \\
\text { Macular Edema }\end{array}$ & $34(23.7 \%)$ & 0.316 \\
\hline
\end{tabular}

\section{Discussion}

OCT seems to be particularly useful for detecting serous retinal detachment, a feature that is consistent with macular edema but that 
is not easily seen on biomicroscopy. It is only after the advent of OCT that this entity has surfaced as a significant finding in many cases of clinically significant macular edema. ${ }^{2}$ The pathogenesis of SRD in DME is still not clear. Kang et al. ${ }^{15}$ postulated that since the external limiting membrane is not impermeable to fluid and albumin therefore with the disruption of the inner blood retinal barrier, the excess fluid might reach the subretinal space in large amounts, might fail to be removed properly by the retinal pigment epithelium (RPE) there by resulting in serous retinal detachment. However, this mechanism does not explain why SRD can occur when DME is focal and far from the macula, and why it can regress before the resolution of DME. Furthermore, as suggested by Gaucher et al. ${ }^{16}$ SRD may appear without large retinal fluid accumulation and its severity does not correlate with foveolar retinal thickness.

In a recent study, ${ }^{17}$ impairment of the choroidal blood flow was reported in patients with DME. The decreased choroidal blood flow may cause tissue hypoxia and RPE function impairment that result in the development of DME. In the study by Gaucher et al. ${ }^{16} 67.5 \%$ of the patients of SRD with DME had uncontrolled hypertension during follow up. Since hypertension is known to impair choroidal circulation and result in serous retinal detachment, they hypothesized that it may be one of the risk factors for the development of SRD in diabetic CSME. In our study, we analysed various systemic and ocular features of 100 consecutive patients of Type 2 diabetes with CSME in at least one eye. The patients were divided into two groups on the basis of presence or absence of SRD on spectral domain OCT (CirrusTM OCT, Zeiss Meditec, Dublin, CA, USA). 30 patients with serous macular detachment in at least one eye were placed in Group 1 and the remaining 70 patients were placed in Group 2. Out of the 177 eyes studied, 42 eyes had serous macular detachment (23.6\%). Previous studies. ${ }^{3,18-20}$ have reported an incidence of $15-30 \%$ of SRD in eyes with DME.

In our study, we found no significant difference in the age of patient, gender or age at onset of diabetes between the two groups of patients. The duration of diabetes was significantly more in Group 2 as compared to Group $1(p=0.02)$ You mean that a longer duration of DM decreases the chance for DME?. None of the drugs for diabetes showed any significant effect on either of the two groups. We could not find any association of history of hypertension, duration of hypertension; mean systolic or diastolic blood pressure with presence of serous macular detachment in diabetic macular edema. A significantly greater number of patients in Group 2 were taking calcium channel blockers $(\mathrm{p}=0.04)$. However, there is no mention of the protective effect of calcium channel blockers in the literature. This means that $\mathrm{Ca}$ channel blockers are protective? If so, mention other studies that share the same result. Rest of the anti-hypertensive medications did not show any significant effect on either of the two groups. There was no significant difference between the two groups in any of the microvascular or macrovascular complications of diabetes such as neuropathy, nephropathy, dialysis, $\mathrm{CAD} / \mathrm{CHF} / \mathrm{PACS} / \mathrm{CABG}$, peripheral vascular disease, diabetic foot etc. Both the groups were matched for weight, height and BMI.

Otani ${ }^{6}$ showed that visual acuity with best correction moderately correlated with retinal thickness regardless of the morphologic pattern observed on OCT. In a retrospective study, Gaucher et al. ${ }^{16}$ showed that SRD height does not correlate with visual acuity. In our study also, there was no significant difference in the BCVA of patients with or without SRD $(\mathrm{P}=0.68)$. In our study, the eyes with SRD had a significantly higher central macular thickness compared to eyes without SRD $(p<0.001)$. However, we did not correlate the height of the SRD in each eye with the central macular thickness or the neuroretinal thickness above the SRD as done by

Moreover, in our study, significantly greater number of eyes with SRD $(40.5 \%)$ were associated with severe NPDR as compared to eyes without SRD $(12.6 \%)(p<0.001)$. No specific pattern on fluorescein angiography was noticed in the eyes with SRD by Gaucher et al. ${ }^{16} \mathrm{In}$ a study by Soliman et al..$^{21}$ the fluorescein angiographic patterns of diabetic macular edema correlated with the patterns of DME seen on OCT except serous macular detachment which did not correlate with any specific pattern on FFA. In our study, the combination of diffuse and cystoid macular edema was the most common FFA pattern seen in $57.1 \%$ of eyes in Group 1 compared to $15.4 \%$ eyes in Group 2 $(p<0.001)$ Group 2 is supposed to be free from SRD. On the other hand, focal macular edema was the most common FFA finding in eyes without SRD and was present in $46.6 \%$ of the eyes in Group 2. In our study, the pattern of DME most commonly associated with SRD on OCT was cystoid macular edema. Cystoid pattern of macular edema was present in a total of 25 eyes (59.5\%) in Group 1 and in 41 eyes $(30.3 \%)$ in Group 2.

The pathophysiologic basis of the above ocular associations of serous retinal detachment in diabetic macular edema are unknown. More prospective longitudinal studies need to be done to determine the natural course of SRD and its response to various treatment modalities currently employed for DME which would help in establishing the underlying pathogenesis of this entity, its correlation with visual acuity as well as any hitherto unknown systemic or ocular factors associated with it. This would further help in better characterisation and effective management of patients with diabetic macular edema.

\section{Funding details}

None.

\section{Acknowledgements}

None.

\section{Conflicts of interest}

Authors declare that there is no conflict of interest.

\section{References}

1. Rudnisky CJ, Hinz BJ, Tennant MT, et al. High-resolution stereoscopic digital fundus photography versus contact lens biomicroscopy for the detection of clinically significant macular edema. Ophthalmology. 2002;109(2):267-274

2. Hirano T, Iesato Y, Toriyama Y, et al. Detection of fovea-threatening diabetic macular edema by optical coherence tomography to maintain good vision by prophylactic treatment. Ophthalmic Research. 2014;52(2):65-73.

3. Virgili G, Menchini F, Murro V, et al. Optical coherence tomography (OCT) for detection of macular oedema in patients with diabetic retinopathy. The Cochrane database of systematic reviews. 2007;7:CD008081.

4. Kinyoun J, Barton F, Fisher M, et al. Detection of diabetic macular edema. Ophthalmoscopy versus photography-Early Treatment Diabetic Retinopathy Study Report Number 5. The ETDRS Research Group. Ophthalmology. 1989;96(6):746-750. 
5. Otani T, Kishi S, Maruyama Y. Patterns of diabetic macular edema with optical coherence tomography. American journal of Ophthalmology. 1999;127(6):688-693.

6. Otani T, Kishi S (2007) Correlation between optical coherence tomography and fluorescein angiography findings in diabetic macular edema. Ophthalmology 114(1):104-107.

7. Yeung L, Lima VC, Garcia P, et al. Correlation between spectral domain optical coherence tomography findings and fluorescein angiography patterns in diabetic macular edema. Ophthalmology. 2009;116(6):11581167.

8. Giocanti-Auregan A, Hrarat L, et al. Functional and Anatomical Outcomes in Patients With Serous Retinal Detachment in Diabetic Macular Edema Treated With Ranibizumab. Invest Ophthalmol Vis Sci. 2017;58(2):797-800

9. Bandello F, Battaglia Parodi M, Lanzetta P, et al. Diabetic Macular Edema. Dev Ophthalmol. 2017;47:73-110.

10. Kiire CA, Porta M, Chong V. Medical management for the prevention and treatment of diabetic macular edema. Survey of Ophthalmology. 2013;58(5):459-465.

11. Ansquer JC, Crimet D, Foucher C. Fibrates and statins in the treatmen of diabetic retinopathy. Curr Pharm Biotechnol. 2011;12(3):396-405.

12. Effects of aspirin treatment on diabetic retinopathy. ETDRS report number 8. Early Treatment Diabetic Retinopathy Study Research Group. Ophthalmology. 1991;98(5 Suppl):757-765.

13. Idris I, Warren G, Donnelly R. Association between thiazolidinedione treatment and risk of macular edema among patients with type 2 diabetes. Arch Intern Med. 2012;172(13):1005-1011.
14. Fundus photographic risk factors for progression of diabetic retinopathy. ETDRS report number 12. Early Treatment Diabetic Retinopathy Study Research Group. Ophthalmology. 1991;98(5 Suppl):823-833.

15. Kang JW, Chung H, Chan Kim H. Correlation Of Optical Coherence Tomographic Hyperreflective Foci With Visual Outcomes In Different Patterns Of Diabetic Macular Edema. Retina. 2016;36(9):1630-1639.

16. Gaucher D, Sebah C, Erginay A, et al. Optical coherence tomography features during the evolution of serous retinal detachment in patients with diabetic macular edema. Am J Ophthalmol. 2008;145(2):289-296.

17. Totan Y, Akyuz TK, Guler E, et al. Evaluation of ocular pulse amplitude and choroidal thickness in diabetic macular edema. Eye (Lond). 2016;30(3):369-374.

18. Ozdemir H, Karacorlu M, Karacorlu S. Serous macular detachment in diabetic cystoid macular oedema. Acta ophthalmologica. 2005;83(1):63-66.

19. Ghosh S, Bansal P, Shejao H, et al. Correlation of morphological pattern of optical coherence tomography in diabetic macular edema with systemic risk factors in middle aged males. Int Ophthalmol. 2015;35(1):3-10.

20. Koleva-Georgieva D, Sivkova N. Assessment of serous macular detachment in eyes with diabetic macular edema by use of spectraldomain optical coherence tomography. Graefes Arch Clin Exp Ophthalmol. 2009;247(11):1461-1469.

21. Soliman W, Sander B, Hasler PW, et al. Correlation between intraretinal changes in diabetic macular oedema seen in fluorescein angiography and optical coherence tomography. Acta Ophthalmol. 2008;86(1):34 39. 